\title{
Implications of Cannabis Use and Heavy Alcohol Use on HIV Drug Risk Behaviors in Russian Heroin Users
}

\author{
Alexander Y. Walley $\cdot$ Evgeny M. Krupitsky • \\ Debbie M. Cheng • Anita Raj · Erika M. Edwards • \\ Carly Bridden · Valentina Y. Egorova $\cdot$ Edwin E. Zvartau • \\ George E. Woody $\cdot$ Jeffrey H. Samet
}

Received: 20 November 2006/Accepted: 16 April 2007/Published online: 9 May 2007

(C) Springer Science+Business Media, LLC 2007

\begin{abstract}
Cannabis and heavy alcohol use potentially increase HIV transmission by increasing risky drug behaviors. We studied 404 subjects entering treatment for heroin dependence, in St. Petersburg, Russia. We used the HIV Risk Assessment Battery (RAB) drug subscale to measure risky drug behavior. Although all heavy alcohol users had risky drug behaviors, their drug RAB scores did not differ from non-heavy alcohol users in unadjusted or adjusted analyses. Cannabis use was significantly associated with drug RAB scores in unadjusted analyses (mean
\end{abstract}

A. Y. Walley $(\varangle) \cdot$ D. M. Cheng · C. Bridden .

J. H. Samet

Clinical Addiction Research and Education (CARE) Unit,

Section of General Internal Medicine, Department of Medicine,

Boston Medical Center and Boston University School of

Medicine, 91 E. Concord St., Suite 200, Boston, MA 02118,

USA

e-mail: awalley@bu.edu

E. M. Krupitsky · V. Y. Egorova · E. E. Zvartau

St. Petersburg Scientific Research Center of Addictions and

Psychopharmacology, St. Petersburg State Pavlov Medical

University, St. Petersburg, Russia

D. M. Cheng

Department of Biostatistics, Boston University School of Public Health, Boston, MA, USA

A. Raj · J. H. Samet

Department of Social and Behavioral Sciences, Boston

University School of Public Health, Boston, MA, USA

E. M. Edwards

Data Coordinating Center, Boston University School of Public

Health, Boston, MA, USA

G. E. Woody

Department of Psychiatry and Veterans Affairs Medical Center, University of Pennsylvania, Philadelphia, PA, USA difference 1.7 points) and analyses adjusted for age, sex, and employment (mean difference 1.3 points). When also adjusting for stimulant use, the impact of cannabis use was attenuated and no longer statistically significant (mean difference 1.1 points). Because of the central role of risky drug behaviors in the Russian HIV epidemic, it is important to understand how the use of multiple substances, including cannabis and alcohol, impacts risky drug behaviors.

Keywords Cannabis - Alcohol · Russia - HIV . Risk behaviors

\section{Introduction}

Since the mid-1990s, HIV infection in the Russian Federation has become the largest HIV epidemic in Europe with an estimated 860,000 (range: 420,000-1.4 million) people living with HIV in 2003 (UNAIDS, 2005). Injection drug use has driven this rapid growth with more than $80 \%$ of officially reported HIV cases occurring among injection drug users (IDUs) (Dehne, Khodakevich, Hamers, \& Schwartlander, 1999; Dehne, Pokrovskiy, Kobyshcha, \& Schwartlander, 2000). Preventing HIV transmission in Russia is challenging because unsafe injection practices, such as sharing needles, are common (Shaboltas et al., 2006), and opioid replacement therapy with methadone or buprenorphine is illegal. Naltrexone is approved and was shown to prevent relapse to heroin use over a 6 -month period in $42-44 \%$ of subjects, but it has not been widely used since its cost $(\$ 3.50 / 50 \mathrm{mg}$ tablet $)$ is prohibitive (Krupitsky et al., 2004b; Krupitsky et al., 2006). With HIV infection rates among IDUs as high as $30 \%$ (Kozlov et al., 2006; Krupitsky et al., 2004a; Shaboltas et al., 2006), the epidemic is crossing over into other risk groups, such as sex workers and sexual partners of injectors (UNAIDS, 2005). 
Russian society has a long history of high levels of alcohol consumption. Nationally representative samples from 1992 to 2004 report about $70 \%$ of men and $45 \%$ of women drink alcohol (Zohoori, Banchette, \& Popkin, 2005). Binge drinking is particularly popular in Russia, where the $20 \%$ of men with the greatest intake average more than $100 \mathrm{~g}$ (i.e., seven drinks) of alcohol per day (McKee, 1999). With an estimated one third of all deaths in Russia in 1994 related to alcohol, alcohol use is a significant Russian public health issue (Nemtsov, 2002; World Health Organization, 2004). Emerging evidence shows active alcohol use among HIV-infected patients in Russia and associations between heavy drinking and HIV risk behaviors (Benotsch et al., 2006; Krupitsky et al., 2005; Somlai et al., 2002).

Little has been published on the potential association between cannabis use and HIV risk behaviors, yet it is the most abused illicit drug in Russia (United Nations Office on Drugs and Crime, 2006). Among 15 and 16 year olds, $5 \%$ are monthly users, $22 \%$ are lifetime users and $24 \%$ report cannabis is easily available (Hibell et al., 2004). Use of other drugs, such as stimulants, has been linked to HIV transmission and risk behaviors in several populations (Buchanan et al., 2006; Lorvick, Martinez, Gee, \& Kral, 2006; Molitor et al., 1999; Pechansky et al., 2006). A recent study among HIV-negative IDUs in St. Petersburg, Russia reports increased HIV seroconversion among IDUs who inject stimulants (Kozlov et al., 2006).

With a rapidly expanding HIV epidemic among IDUs, in the setting of high levels of cannabis and alcohol consumption, it is important to determine whether cannabis and alcohol use impacts risky drug behaviors. If HIV transmission is increased by cannabis or alcohol consumption, efforts to reduce cannabis or alcohol use may be effective HIV prevention strategies. We examined the association of cannabis use and alcohol use with HIV drugrisk behaviors in Russian drug users in this secondary analysis of baseline data from three randomized control trials conducted between 1999 and 2005.

\section{Methods}

\section{Participants}

We studied 404 patients undergoing treatment for heroin dependence in and around St. Petersburg. Between 1999 and 2002, 332 of these subjects were recruited into two randomized controlled trials of naltrexone for relapse prevention (Krupitsky et al., 2004b; Krupitsky et al., 2006). Trained physician research associates recruited these subjects during inpatient treatment at addiction hospitals affiliated with the St. Petersburg Pavlov State Medical
University or by referral from community psychiatrists after outpatient detoxification. Eligibility criteria for these two trials were identical: a diagnosis of heroin dependence; age between 18 and 40 years; education at the high school level or above; at least one relative willing to support the subject's treatment and supervise adherence to study medications; a stable address with a working phone; no regular use of psychiatric medication; a negative pregnancy test and commitment to contraceptive use, if female; and abstinence from heroin and other substances for at least one week prior to study entry.

The remaining 72 subjects were the heroin dependent subgroup of the Russian PREVENT (Partnership to Reduce the Epidemic Via Engagement in Narcology Treatment) study. PREVENT was a randomized controlled trial of an HIV prevention intervention with the goal of reducing HIV sexual risk behaviors (Samet et al., 2006). PREVENT subjects were enrolled in 2004 and 2005. Trained physician research associates approached patients at the Leningrad Regional Center for Addictions and the Medical Narcology Rehabilitation Center after initial detoxification, and assessed eligibility, offered participation, and conducted assessments. Eligibility criteria included age 18 years and older; no alcohol or other abused substances for at least $48 \mathrm{~h}$; reported unprotected anal or vaginal sex in the past 6 months; willingness to undergo HIV testing per standard narcology hospital protocols or previous diagnosis of HIV infection; and provision of reliable contact information (i.e., a home telephone number, an address within $150 \mathrm{~km}$ of St. Petersburg, and a friend or family contact).

In the current analysis, we combined these cohorts, as subjects were recruited from the same geographic area, under similar conditions, and with similar entry criteria and were assessed with similar baseline study instruments and methods. All subjects provided written informed consent prior to enrollment in the studies.

\section{Measures}

Baseline assessment in each cohort included the following: demographic survey; the Risk Assessment Battery (RAB) to assess HIV drug risk behavior in the past 6 months (Metzger et al., 1992; Metzger, Navaline, \& Woody, 2001; Navaline et al., 1994); and the 30-day Timeline Followback (TLFB) survey for alcohol and cannabis use (FalsStewart, O'Farrell, Freitas, McFarlin, \& Rutigliano, 2000; Sobell \& Sobell, 1992; Sobell \& Sobell, 1995). For the Naltrexone cohort, stimulant use was assessed using the 30-day TLFB. We categorized subjects with any stimulant use in the 30 days prior as stimulant users. For the PREVENT cohort, stimulant use in the last 30 days was assessed using the Addiction Severity Index (ASI) (McLellan et al., 1985). The ASI includes questions about 
cocaine and amphetamine use in the past 30 days. Those subjects who answered yes to either of these questions were categorized as stimulant users. All study instruments were translated into Russian and checked for clarity by Russian research staff. Trained research staff delivered all survey instruments in one-on-one confidential sessions, except in the Russian PREVENT cohort, where the RAB was administered by an Audio Computer-Assisted SelfInterviewing (ACASI) System. ACASI allows additional privacy, minimizes literacy issues, encourages truth telling, and provides an identical recording of each question. Using this system has been shown to enhance the quality of selfreport behavioral assessments and to provide an acceptable method for collecting self-reports of HIV risk behavior (Newman et al., 2002). HIV infection was recorded as part of the intake assessment for the PREVENT cohort, but not for the naltrexone cohorts. Subjects in all cohorts were compensated US\$ 5 for participation in the baseline assessment.

Main Independent Variables

\section{Heavy Alcohol Use}

The TLFB survey asked subjects to recall the amount of alcohol consumed on each of the 30 days prior to entering treatment. From these data on their average weekly intake, alcohol status was classified into the following two categories: heavy (>seven drinks/week for women and >14/ week for men) and not heavy (alcohol use less than heavy thresholds). We chose these categories to be consistent with National Institute on Alcohol Abuse and Alcoholism recommendations for heavy drinking. There are no established heavy drinking limits in Russia.

\section{Cannabis Use}

The TLFB survey in all cohorts asked subjects to recall cannabis use on each of the 30 days prior to entering treatment. We categorized subjects with any cannabis use in the 30 days prior as a cannabis user and those with no cannabis use as a cannabis non-user.

\section{Outcomes}

\section{Measures of HIV Drug-Risk Behavior}

The primary outcome of the study was the drug RAB score, modeled as a continuous variable. The drug RAB score ranges from 0 to 25 . Injecting drugs within the last 6 months scored 1 point. Additional points were scored for needle sharing, equipment sharing, injecting in group settings, and mixing and sharing drugs. Among a prospectively followed group of IDUs in the United States, the drug RAB was able to discriminate those who seroconverted from those who remained seronegative for HIV infection (Metzger et al., 2001). A secondary outcome was risky drug use in the past 6 months. We defined risky drug use as having a drug RAB score greater than 1 , which indicates some needle, equipment, or drug sharing in the last 6 months.

\section{Data Analysis}

We performed descriptive analyses (e.g. means, medians, standard deviations, proportions) for the following variables: age, sex, employment status, HIV status (available for PREVENT study only), stimulant use, cannabis use, heavy alcohol use, mean drug RAB score, and risky drug use. Subjects in the two naltrexone trials were grouped into the Naltrexone cohort $(n=332)$ and compared to the PREVENT cohort $(n=72)$ using two-sample $t$-tests or $\chi^{2}$ tests. We assessed whether heavy alcohol use and cannabis use were associated with drug RAB score using multiple linear regression models, adjusting for age, sex, employment status, stimulant use and study cohort as covariates. Logistic regression models were used to analyze the secondary dichotomous outcome, risky drug use. To assess whether the effect of heavy alcohol use and cannabis use was similar for both cohorts, regression models were also fit separately for each cohort. For the PREVENT cohort, we performed an additional model that included a covariate for HIV status. All analyses were conducted using twosided tests and a significance level of 0.05 .

\section{Results}

Characteristics of the combined and individual cohorts are presented in Table 1. The combined cohort included $27 \%$ women, $28 \%$ employed, $13 \%$ stimulant users, $10 \%$ drinking at heavy levels, and $91 \%$ with a drug RAB score greater than 1. The Naltrexone and PREVENT cohorts were similar overall, but the PREVENT cohort was older (27 years vs. $23 ; t(402)=5.81 ; P<0.01)$; had more cannabis users $\left(33 \%\right.$ vs. $22 \% ; \chi^{2}(1, n=404)=4.06$; $P<0.05$ ); and had a higher mean drug RAB score (12.6 vs. $8.0 ; t(402)=6.86 ; P<0.01)$. HIV infection status was only known for the PREVENT cohort, in which $35 \%$ were HIV-infected. The cohorts were similar with regards to gender, employment, stimulant use and heavy alcohol use. When stratified by heavy alcohol use, characteristics were similar for heavy and non-heavy alcohol users. When stratified by cannabis use, cannabis and non-cannabis users were similar on all characteristics except cannabis users were more likely to use stimulants $\left(22 \%\right.$ vs. $10 \% ; \chi^{2}(1$, $n=404)=9.96 ; P<0.01)$. 
Table 1 Characteristics of IDUs in St. Petersburg overall and stratified by study cohort

\begin{tabular}{|c|c|c|c|c|}
\hline & Total $n=404$ & PREVENT $n=72$ & Naltrexone $n=332$ & Test statistic ${ }^{\mathrm{a}}$ \\
\hline Age, mean (SD) & $23.9(4.5)$ & $26.6(4.9)$ & $23.4(4.2)$ & $5.81 * *$ \\
\hline Male, no. (\%) & $294(73)$ & $49(68)$ & $245(74)$ & 0.98 \\
\hline Employed, no. (\%) & $111(28)$ & $18(25)$ & $93(28)$ & 0.27 \\
\hline \multicolumn{5}{|l|}{ HIV status, no. (\%) } \\
\hline Positive & $25(6)$ & $25(35)$ & 0 & NA \\
\hline Negative & $47(12)$ & $47(65)$ & 0 & \\
\hline Unknown & $332(82)$ & 0 & $332(100)$ & \\
\hline Stimulant use, no. (\%) & $51(13)$ & $12(17)$ & $39(12)$ & 1.28 \\
\hline Cannabis use, no. (\%) & $97(24)$ & $24(33)$ & $73(22)$ & $4.06^{*}$ \\
\hline Heavy alcohol use no. (\%) & $40(10)$ & $7(10)$ & $33(10)$ & 0.0051 \\
\hline Risky IDU, no. (\%) & $368(91)$ & $67(93)$ & $301(91)$ & 0.42 \\
\hline Mean drug RAB score (SD) & $8.9(5.4)$ & $12.6(6.3)$ & $8.0(4.9)$ & $6.86^{* *}$ \\
\hline
\end{tabular}

$* P<0.05, * * P<0.01$

a $t$-tests $($ degrees of freedom $=402)$ and $\chi^{2}$ tests $($ degrees of freedom $=1)$

Table 2 displays the bivariate analyses of cannabis use and heavy alcohol use with drug RAB score and risky drug use. In unadjusted analyses, cannabis users had a higher mean drug RAB score compared to non-users (10.2 vs. 8.5; $t(402)=2.74 ; P<0.01)$, however there was no difference in the proportion of risky drug use $\left(93 \%\right.$ vs. $91 \% ; \chi^{2}(1$, $n=404)=0.71$; ns). We did not find a significant difference in drug RAB score between heavy and non-heavy drinkers $(9.6$ vs. $8.8 ; t(402)=0.89$; ns), however, heavy drinkers were more likely to be risky IDUs (100\% versus 90\%; Fisher's exact test; $P<0.05$ ), as defined above in "Outcomes".

In multiple regression analyses, the mean drug $\mathrm{RAB}$ score remained higher for cannabis users compared to noncannabis users (adjusted mean difference 1.09 points; $t(402)=1.82$; ns; Table 3 ), however the magnitude of the association was attenuated and no longer statistically significant. Because cannabis use has been described as a gateway drug, and therefore may precede stimulant use in the causal pathway, we performed an adjusted analysis that did not include stimulant use which showed cannabis users had a mean drug RAB score 1.25 points higher than noncannabis users $(t(402)=2.10 ; P<0.05)$. No statistically significant association between heavy alcohol use and the continuous drug RAB score was detected in both the model adjusted for stimulant use (adjusted mean difference in drug RAB score 0.59 for heavy versus non-heavy alcohol use; $t(402)=0.71 ; \mathrm{ns}$ ) and the model that was not (adjusted mean difference $0.63 ; t(402)=0.76 ; \mathrm{ns})$. Significant associations with lower drug RAB scores among covariates included older age, current employment, and participation in the Naltrexone cohort.

In secondary multiple linear regression analyses stratified by study cohort, associations between cannabis use and higher drug RAB scores were observed for both cohorts; however, the associations were not statistically significant. In both study cohorts, older age was significantly associated with lower drug RAB scores. Current employment was associated with lower RAB scores, though statistically significant for the PREVENT cohort, but not the Naltrexone cohort. In the PREVENT cohort analysis that included HIV status, subjects with HIV infection had higher drug RAB scores than those who were not HIV-infected (the mean drug RAB score was 4.6 points higher for HIV positive versus HIV negative subjects, $t(70)=3.26$; $P<0.01)$.

Table 2 Bivariate comparisons of heavy alcohol use, cannabis use with drug risk behaviors

\begin{tabular}{lcccc}
\hline & Mean drug RAB score (SD) & T-test statistic $(\mathrm{df}=400)$ & Risky IDU, no. $(\%)$ & $\chi^{2}$ statistic $(\mathrm{df}=1)$ \\
\hline Heavy alcohol use & $9.6(5.3)$ & -0.89 & $40(100)$ & $*, \mathrm{a}$ \\
No heavy alcohol use & $8.8(5.4)$ & & $326(90)$ & \\
Cannabis use & $10.2(5.5)$ & $2.74 * *$ & $90(93)$ & 0.47 \\
No cannabis use & $8.5(5.3)$ & & $276(91)$ & \\
\hline
\end{tabular}

$* P<0.05, * * P<0.01$

a Calculated using Fisher's exact test 
Table 3 Multiple linear regression models assessing the impact of heavy alcohol use and cannabis use on drug RAB score among IDUs in St. Petersburg $(n=404)$

\begin{tabular}{|c|c|c|c|c|c|c|}
\hline & \multicolumn{3}{|c|}{ Adjusted for stimulant use } & \multicolumn{3}{|c|}{ Not adjusted for stimulant use } \\
\hline & $\begin{array}{l}\text { Mean change } \\
\text { in drug RAB }\end{array}$ & Standard error & $T$-value & $\begin{array}{l}\text { Mean change } \\
\text { in drug RAB }\end{array}$ & Standard error & $T$-value \\
\hline Heavy alcohol use & +0.59 & 0.84 & 0.71 & +0.63 & 0.84 & 0.76 \\
\hline Cannabis use & +1.09 & 0.60 & 1.82 & +1.25 & 0.59 & $2.10^{*}$ \\
\hline $\mathrm{Age}^{\mathrm{a}}$ & -0.23 & 0.059 & $-4.07 * *$ & -0.24 & 0.059 & $-4.15^{* *}$ \\
\hline Female vs. Male & -0.30 & 0.57 & -0.52 & -0.23 & 0.57 & -0.41 \\
\hline Employed & -1.47 & 0.56 & $-2.63 * *$ & -1.53 & 0.56 & $-2.74 * *$ \\
\hline Naltrexone vs. PREVENT cohort & -5.13 & 0.68 & $-7.52 * *$ & -5.19 & 0.68 & $-7.60 * *$ \\
\hline Stimulant use & +1.28 & 0.77 & 1.67 & $\mathrm{X}$ & $\mathrm{X}$ & $\mathrm{X}$ \\
\hline
\end{tabular}

* $P<0.05, * * P<0.01$

a Adjusted mean corresponding to a 1 year increase in age

Unadjusted and adjusted logistic regression models showed no significant association between cannabis use and the secondary outcome risky drug use (unadjusted Odds Ratio $1.35 ; 95 \%$ confidence interval $0.57-3.19 ; \chi^{2}(1$, $n=402)=0.47$; ns; adjusted Odds Ratio 1.30 ; $95 \%$ confidence interval $\left.0.53-3.17 ; \chi^{2}(1, n=402)=0.32 ; \mathrm{ns}\right)$. In logistic regression models including heavy alcohol use as an independent variable, odds ratios were not estimable as all heavy drinkers were risky drug users.

\section{Discussion}

Reducing drug-related HIV transmission in Russia will require wide adoption by the existing public health systems of effective prevention and treatment efforts, such as needle exchange programs, increased access to naltrexone and availability of methadone or buprenorphine replacement programs. Understanding the relationship between substance use (e.g., alcohol, cannabis, and stimulants) and injection drug use practices should inform the implementation of these programs. We hypothesized that among heroin dependent patients entering treatment in Russia, both heavy alcohol use and cannabis use would be independently associated with risky drug use behaviors. In unadjusted analysis, we found a statistically significant increase in drug RAB score among cannabis users. But in a multiple regression analysis this association was attenuated and not statistically significant after adjustment for stimulant use. We did not detect a significant association between heavy alcohol use and drug RAB score, yet we found that all heavy alcohol users had some needle, equipment, or drug sharing in the last 6 months.

How could cannabis use increase HIV drug risk behaviors? Research focused on the relationship of cannabis use and HIV drug risk behaviors is limited. Cannabis use is associated with the use of other drugs and frequently precedes the use of injectable drugs, such as heroin and cocaine (Fergusson, Boden, \& Horwood, 2006; Golub \& Johnson, 1994; Lynskey et al., 2003). A French cohort study of HIV-infected IDUs found that cessation of injection drug use was associated with decreased cannabis use (Bouhnik et al., 2004). No studies have directly assessed the hypothesis that cannabis use is associated with risky drug behaviors, either directly via immediate disinhibitory effects or as a marker of risky behavior.

Kozlov et al. reported increased incidence of HIV seroconversion among IDUs in St. Petersburg who use stimulants (Kozlov et al., 2006). Incidence was further increased with greater weekly injection frequency, however this study did not examine the impact of other drugs, such as alcohol or cannabis. It is possible that an association between heavy alcohol use, cannabis use and increased risk behaviors, as well as stimulant use and increased HIV seroconversion are examples of polysubstance use driving increased risk taking. In our adjusted model that did not include stimulant use, we found a significant association between cannabis use and increased drug RAB score, yet this association was attenuated and not significant in the model that included stimulant use. This attenuation may occur because cannabis use is a marker of one or more factors, such as stimulant use, which increases risk taking. Cannabis may be used concomitantly to attenuate the dysphoric results of stimulant use, or cannabis use may precede stimulant use in a causal pathway where cannabis use leads to stimulant use and then to increased risk taking.

The impact of cannabis use on HIV sex risk behaviors has been described (Brodbeck, Matter, \&, Moggi, 2006; Simbayi et al., 2004; Somlai et al., 2002; Woody et al., 1999) but results have not always been inconsistent. Woody et al. (1999) found that cannabis use was not associated with increased sex risk among gay men, while 
Brodbeck et al. (2006) found in a random sample of Swiss heterosexuals that cannabis use was associated with increased HIV sex risk behaviors, though not specifically at the time they were using cannabis. This is evidence that cannabis use is a marker of a riskier personality, or increases risk via chronic effects rather than directly increasing sex risk during the time it is used. In addition, chronic use may affect general risk taking by lowering motivation to protect oneself. If the effect of cannabis use is primarily an increase in general risk taking rather than acute situational risk taking, its use likely increases both HIV drug and sexual behaviors through a similar mechanism. HIV risk behavior research that addresses the impact of acute and chronic cannabis use on measures of motivation might further clarify the mechanism by which cannabis may increase risk behaviors.

Results of studies on alcohol's relationship to HIV risk among IDUs in the United States are mixed. Alcohol use among needle exchange participants in Providence, RI has been associated with increased injection and sexual risk (Stein et al., 2000). Using 30-day TLFB for both risky injection drug use and alcohol use, an association has been shown between daily alcohol use and daily risky injection (Stein, Charuvastra, Anderson, Sobota, \& Friedmanna, 2002). Among Puerto Rican IDUs not in treatment, alcohol intoxication has been associated with sharing needles and cotton filters (Matos et al., 2004). However, among detoxification inpatients in Boston, alcohol consumption was associated with increased sexual risk, but not injection risk (Rees, Saitz, Horton, \& Samet, 2001). Studies in Russia have shown that among HIV-infected inpatients, alcohol abuse or dependence has a significant association with sexual risk and a non-significant association with injection risk (Krupitsky et al., 2005). Among young injection drug users in St. Petersburg, Somlai et al. (2002) reported a $40 \%$ rate of needle sharing in the previous 90 days and a $74 \%$ rate of alcohol use in the previous 30 days, but this study did not look specifically at the association between alcohol and risky injection.

Alcohol and drug use disorders often co-occur in US populations (Belenko, 1979; Kessler et al., 1997), yet these Russian IDUs had a low percentage (10\%) of heavy drinking. With the high overall prevalence of alcohol use in Russia, we expected to find higher rates of alcohol use among heroin users. The low proportion of heavy drinkers in these samples limited our ability to detect a significant association with risky drug behaviors. In unadjusted analyses, we found an association between heavy drinking and the secondary dichotomous outcome, risky drug use, but we were unable to estimate odds ratios from logistic regression models as all heavy drinkers also had risky drug behaviors. We did not find an association between heavy drinking and the continuous drug RAB score, however the measure of heavy drinking we used was developed in the United States and may not be the optimal measure in Russia. Because Russians consume more alcohol than Americans, it is conceivable that the optimal Russian measure may be a higher threshold, which in this study would have the impact of categorizing even fewer heavy drinkers. Despite the absence of a statistically significant association, we caution that alcohol may still represent an important predictor of HIV drug risk behaviors. As risky drug practices in Russia improve with more prevention education and optimal measures of heavy alcohol use in Russia are developed, a relationship between alcohol use and risky injection drug use may be revealed, as the heavy drinkers may be slow adopters of safer injection behaviors. Alternatively, the impact of heavy alcohol use may be limited to sexual risk behaviors.

The association of HIV infection with increased drug RAB scores observed in the PREVENT cohort is consistent with the rapid spread of HIV among IDUs in Russia. Furthermore, the mean drug RAB scores in both cohorts were higher than those seen among drug using Russian (Krupitsky et al., 2005) and American (Rees et al., 2001; Stein et al., 2000) cohorts and may well reflect increased risky drug behavior in Russia. The higher means among the PREVENT cohort relative to the Naltrexone cohort may be explained by the data collection methods. In PREVENT, drug risk behaviors were assessed with the use of the ACASI system which likely reduces social desirability bias and increases truth telling, whereas the Naltrexone cohort was assessed with one-on-one interviews, similar to the other studies that have used the RAB instrument.

There are several limitations to our study. First, our analysis was potentially underpowered to detect effects of the observed magnitude for cannabis and heavy alcohol use. Post-hoc calculations indicate that our study would have approximately $80 \%$ power to detect a minimum difference in drug RAB score of 2.5 and 1.8 for heavy drinking and cannabis use, respectively. Thus, it is likely that the study was not sufficiently powered to detect the observed magnitudes of association. Second, this is a crosssectional analysis of baseline data, which limits our ability to establish causality, as well as the order of preceding causal elements. Third, this study combines results from three different cohorts that enrolled subjects at different times. However, the subjects were recruited and enrolled by similar study staff from similar settings and provided similar data. Fourth, while we did adjust for age, gender, and employment, future research should examine the impact of other psychosocial factors, such as mental illness on drug-related HIV risk behavior. Fifth, as all subjects came from the St. Petersburg area and were entering treatment, they may not adequately represent all Russian IDUs. IDUs entering treatment are likely to be more motivated to re- 
duce not only their heroin use, but their alcohol and cannabis use as well. Therefore it is possible that they may have fewer drug-related risk behaviors before entering treatment. If this were the case, then this would decrease the likelihood we would find an association. Sixth, although we had cannabis use and heavy alcohol use data via the 30-day TLFB method, our risk behavior data was limited to the drug RAB score, which encompasses the prior 6 months. Collecting drug and alcohol use information as well as drug behavior information on a day-by-day basis would allow for examination of the daily relationship between substance use and risky behaviors.

Among 404 Russian IDUs entering treatment for heroin dependence, all heavy alcohol users had risky drug behaviors, but we did not find significant evidence of an association between heavy alcohol use and the HIV drug RAB score in unadjusted or adjusted analyses. We did find a significant association between cannabis use and drug RAB score after adjustment for age, sex, and employment status. Yet when adjusted for stimulant use, the association between cannabis use and HIV drug risk behaviors was attenuated and not statistically significant. Because of the central role of IDUs in the rapidly progressing Russian HIV epidemic, it is important to understand how the use of multiple substances, including cannabis and alcohol, impacts risky drug behaviors among IDUs.

Acknowledgments This work was supported in part by the National Institute on Alcohol Abuse and Alcoholism (NIAAA), NIH: R21AA014821; NIDA U10-DA13043 and K05-DA 17009 (Dr. Woody). Dr. Walley was supported by National Institute on Drug Abuse (NIDA), NIH: R25-DA13582 and National Institute of Allergy and Infectious Diseases (NIAID), NIH: T32-AI52074 04. Dr. Samet is also supported by NIAAA: K24-AA015674. We would also like to acknowledge the assistance and support of Seville Meli and Suzette Levenson in the data collection and conceptualization of this project.

\section{Reference}

Belenko, S. (1979). Alcohol abuse by heroin addicts: Review of research findings and issues. International Journal of Addictions, 14, 965-975.

Benotsch, E. G., Pinkerton, S. D., Dyatlov, R. V., DiFranceisco, W., Smirnova, T. S., Dudko, V. Y., \& Kozlov, A. (2006). HIV risk behavior in male and female Russian sexually transmitted disease clinic patients. International Journal of Behavioral Medicine, 13, 26-33.

Bouhnik, A. D., Carrieri, M. P., Rey, D., Spire, B., Gastaut, J. A., Gallais, H., \& Obadia, Y. (2004). Drug injection cessation among HIV-infected injecting drug users. Addictive Behaviors, 29, 1189-1197.

Brodbeck, J., Matter, M., \& Moggi, F. (2006). Association between cannabis use and sexual risk behavior among young heterosexual adults. AIDS and Behavior, 10, 599-605.

Buchanan, D., Tooze, J. A., Shaw, S., Kinzly, M., Heimer, R., \& Singer, M. (2006). Demographic, HIV risk behavior, and health status characteristics of "crack" cocaine injectors compared to other injection drug users in three New England cities. Drug and Alcohol Dependence, 81, 221-229.

Dehne, K. L., Khodakevich, L., Hamers, F. F., \& Schwartlander, B. (1999). The HIV/AIDS epidemic in eastern Europe: recent patterns and trends and their implications for policy-making. AIDS, 13, 741-749.

Dehne, K. L., Pokrovskiy, V., Kobyshcha, Y., \& Schwartlander, B. (2000). Update on the epidemics of HIV and other sexually transmitted infections in the newly independent states of the former Soviet Union. AIDS, 14(Suppl 3), S75-S84.

Fals-Stewart, W., O'Farrell, T. J., Freitas, T. T., McFarlin, S. K., \& Rutigliano, P. (2000). The timeline followback reports of psychoactive substance use by drug-abusing patients: psychometric properties. Journal of Consulting and Clinical Psychology, 68, 134-144.

Fergusson, D. M., Boden, J. M., \& Horwood, L. J. (2006). Cannabis use and other illicit drug use: Testing the cannabis gateway hypothesis. Addiction, 101, 556-569.

Golub, A., \& Johnson, B. D. (1994). The shifting importance of alcohol and marijuana as gateway substances among serious drug abusers. Journal of Studies on Alcohol, 55, 607-614.

Hibell, B., Andersson, B., Bjarnason, T., Ahlstrom, S., Balakireva, O., Kokkevi, A., \& Morgan, M. (2004). The ESPAD Report 2003: Alcohol and other drug use among students in 35 European countries. Retrieved August 8, 2006, from http://www2.stakes.fi/ english/publications_2000-2004/Kirjat\%202004/m204.htm.

Kessler, R. C., Crum, R. M., Warner, L. A., Nelson, C. B., Schulenberg, J., \& Anthony, J. C. (1997). Lifetime co-occurrence of DSM-III-R alcohol abuse and dependence with other psychiatric disorders in the National Comorbidity Survey. Archives of General Psychiatry, 54, 313-321.

Kozlov, A. P., Shaboltas, A. V., Toussova, O. V., Verevochkin, S. V., Masse, B. R., Perdue, T., Beauchamp, G., Sheldon, W., Miller, W. C., Heimer, R., Ryder, R. W., \& Hoffman, I. F. (2006). HIV incidence and factors associated with HIV acquisition among injection drug users in St Petersburg, Russia. AIDS, 20, 901-906.

Krupitsky, E., Zvartau, E., Karandashova, G., Horton, N. J., Schoolwerth, K. R., Bryant, K., \& Samet, J. H. (2004a). The onset of HIV infection in the Leningrad region of Russia: A focus on drug and alcohol dependence. HIV Medicine, 5, 30-33.

Krupitsky, E. M., Horton, N. J., Williams, E. C., Lioznov, D., Kuznetsova, M., Zvartau, E., \& Samet, J. H. (2005). Alcohol use and HIV risk behaviors among HIV-infected hospitalized patients in St. Petersburg, Russia. Drug and Alcohol Dependence, 79, 251-256.

Krupitsky, E. M., Zvartau, E. E., Masalov, D. V., Tsoi, M. V., Burakov, A. M., Egorova, V. Y., Didenko, T. Y., Romanova, T. N., Ivanova, E. B., Bespalov, A. Y., Verbitskaya, E. V., Neznanov, N. G., Grinenko, A. Y., O'Brien, C. P., \& Woody, G. E. (2004b). Naltrexone for heroin dependence treatment in St. Petersburg, Russia. Journal of Substance Abuse Treatment, 26, 285-294.

Krupitsky, E. M., Zvartau, E. E., Masalov, D. V., Tsoi, M. V., Burakov, A. M., Egorova, V. Y., Didenko, T. Y., Romanova, T. N., Ivanova, E. B., Bespalov, A. Y., Verbitskaya, E. V., Neznanov, N. G., Grinenko, A. Y., O’Brien, C. P., \& Woody, G. E. (2006). Naltrexone with or without fluoxetine for preventing relapse heroin addiction treatment in St. Petersburg, Russia. Journal of Substance Abuse Treatment, 31, 319-328.

Lorvick, J., Martinez, A., Gee, L., \& Kral, A. H. (2006). Sexual and injection risk among women who inject methamphetamine in San Francisco. Journal of Urban Health, 83, 497-505.

Lynskey, M. T., Heath, A. C., Bucholz, K. K., Slutske, W. S., Madden, P. A., Nelson, E. C., Statham, D. J., \& Martin, N. G. (2003). Escalation of drug use in early-onset cannabis users vs 
co-twin controls. Journal of the American Medical Association, 289, 427-433.

Matos, T. D., Robles, R. R., Sahai, H., Colon, H. M., Reyes, J. C., Marrero, C. A., Calderon, J. M., \& Shepard, E. W. (2004). HIV risk behaviors and alcohol intoxication among injection drug users in Puerto Rico. Drug and Alcohol Dependence, 76, 229234.

McKee, M. (1999). Alcohol in Russia. Alcohol and Alcoholism, 34, $824-829$.

McLellan, A. T., Luborsky, L., Cacciola, J., Griffith, J., Evans, F., Barr, H. L., \& O'Brien, C. P. (1985). New data from the Addiction Severity Index. Reliability and validity in three centers. Journal of Nervous and Mental Disease, 173, 412-423.

Metzger, D. S., DePhillips, D., Druley, P., O’Brien, C. P., McLellan, A. T., Williams, J., Navaline, H., Dyanick, S., \& Woody, G. E. (1992). The impact of HIV testing on risk for AIDS behaviors. NIDA Research Monograph, 119, 297-298.

Metzger, D. S., Navaline, H. A., \& Woody, G. E. (2001). Assessment of Substance Abuse: HIV Risk Assessment Battery. In D. Carson (Ed.), Encyclopedia of drugs, alcohol and addictive behavior. Farmington Mills, Michigan: Macmillan Reference USA.

Molitor, F., Ruiz, J. D., Flynn, N., Mikanda, J. N., Sun, R. K., \& Anderson, R. (1999). Methamphetamine use and sexual and injection risk behaviors among out-of-treatment injection drug users. American Journal of Drug and Alcohol Abuse, 25, 475493.

Navaline, H. A., Snider, E. C., Petro, C. J., Tobin, D., Metzger, D., Alterman, A. I., \& Woody, G. E. (1994). Preparations for AIDS vaccine trials. An automated version of the Risk Assessment Battery (RAB): Enhancing the assessment of risk behaviors. AIDS Research and Human Retroviruses, 10(Suppl 2), S281S283.

Nemtsov, A. V. (2002). Alcohol-related human losses in Russia in the 1980s and 1990s. Addiction, 97, 1413-1425.

Newman, J. C., Des Jarlais, D. C., Turner, C. F., Gribble, J., Cooley, P., \& Paone, D. (2002). The differential effects of face-to-face and computer interview modes. American Journal of Public Health, 92, 294-297.

Pechansky, F., Woody, G., Inciardi, J., Surratt, H., Kessler, F., Von Diemen, L., \& Bumaguin, D. B. (2006). HIV seroprevalence among drug users: an analysis of selected variables based on 10 years of data collection in Porto Alegre, Brazil. Drug and Alcohol Dependence, 82(Suppl 1), S109-S113.

Rees, V., Saitz, R., Horton, N. J., \& Samet, J. (2001). Association of alcohol consumption with HIV sex- and drug-risk behaviors among drug users. Journal of Substance Abuse Treatment, 21, $129-134$

Samet, J. H., Krupitsky, E., Cheng, D. M., Raj, A., Egorova, V., Levenson, S., Meli, S., Bridden, C., Verbitskaya, E., Kamb, M. L., \& Zvartau, E. (2006, August). A randomized controlled trial (RCT) to reduce HIV sex-risk behaviors among hospitalized Russian substance dependent patients: The Russian Partnership to Reduce the Epidemic Via Engagement in Narcology Treat- ment (Russian PREVENT) Study. Poster session presented at the XVI International AIDS Conference, Toronto, Canada.

Shaboltas, A. V., Toussova, O. V., Hoffman, I. F., Heimer, R., Verevochkin, S. V., Ryder, R. W., Khoshnood, K., Perdue, T., Masse, B. R., \& Kozlov, A. P. (2006). HIV prevalence, sociodemographic, and behavioral correlates and recruitment methods among injection drug users in St. Petersburg, Russia. Journal of Acquired Immune Deficiency Syndromes, 41, 657663.

Simbayi, L. C., Kalichman, S. C., Jooste, S., Mathiti, V., Cain, D., \& Cherry, C. (2004). Alcohol use and sexual risks for HIV infection among men and women receiving sexually transmitted infection clinic services in Cape Town, South Africa. Journal of Studies on Alcohol, 65, 434-442.

Sobell, L., \& Sobell, M. (1992). Timeline followback: a technique for assessing self-reported alcohol consumption. In R. Z. Litten \& J. P. Allen (Eds.), Measuring alcohol consumption: Psychosocial and biological methods (pp. 41-72). Totawa, NJ: The Humana Press Inc.

Sobell, L., \& Sobell, M. (1995). Alcohol timeline followback (TLFB) Users' Manual. Toronto, Canada: Addiction Research Foundation.

Somlai, A. M., Kelly, J. A., Benotsch, E., Gore-Felton, C., Ostrovski, D., McAuliffe, T., \& Kozlov, A. P. (2002). Characteristics and predictors of HIV risk behaviors among injection-drug-using men and women in St. Petersburg, Russia. AIDS Education and Prevention, 14, 295-305.

Stein, M. D., Charuvastra, A., Anderson, B., Sobota, M., \& Friedmanna, P. D. (2002). Alcohol and HIV risk taking among intravenous drug users. Addictive Behaviors, 27, 727-736.

Stein, M. D., Hanna, L., Natarajan, R., Clarke, J., Marisi, M., Sobota, M., \& Rich, J. (2000). Alcohol use patterns predict high-risk HIV behaviors among active injection drug users. Journal of Substance Abuse Treatment, 18, 359-363.

UNAIDS (2005). AIDS epidemic update: December 2005. Retrieved August 8, 2006, from http://www.unaids.org/epi/2005/doc/EPIupdate2005_pdf_en/epi-update2005_en.pdf.

United Nations Office on Drugs and Crime (2006). 2006 World drug report. Retrieved August 8, 2006, from www.unodc.org/unodc/ world_drug_report.html.

World Health Organization (2004). Global status report on alcohol 2004: Russian Federation. Retrieved August 8, 2006, from http:// www.who.int/substance_abuse/publications/alcohol/en/.

Woody, G. E., Donnell, D., Seage, G. R., Metzger, D., Marmor, M., Koblin, B. A., Buchbinder, S., Gross, M., Stone, B., \& Judson, F. N. (1999). Non-injection substance use correlates with risky sex among men having sex with men: Data from HIVNET. Drug and Alcohol Dependence, 53, 197-205.

Zohoori, N., Banchette, D., \& Popkin, B. (2005). Monitoring Health Conditions in the Russian Federation: The Russia Longitudinal Monitoring Survey 1992-2004. Report submitted to the U.S. Agency for international development. Retrieved August 8, 2006, from http://www.cpc.unc.edu/projects/rlms/papers.html. 